\title{
Evaluation of Systematic Error and Uncertainty of Viscosity Measurements of Mixtures of Monoethanol Amine and Water in Coaxial Cylinder Rheometers
}

\author{
Sumudu S. Karunarathne, Dag A. Eimer, and Lars E. Øi
}

\begin{abstract}
In this study, the use of coaxial cylinder viscometer in viscosity measurements of monoethanol amine and water mixture is discussed. Random and systematic effects engage with the rheometer lead to deviate the measured quantity from its actual value. Compensation for the systematic effect is called as the bias and this compensation can not be done perfectly. The measurement uncertainty arises due to the lack of exact knowledge on what is being measured. Identification of uncertainty sources is vital in uncertainty analysis to evaluate the total uncertainty of a measuring technique. The calculated expanded $(k=2)$ uncertainty of viscosity measurement of an alkanol amine and water mixture using a coaxial cylinder viscometer in this work is $0.0162 \mathrm{mPa} \cdot \mathrm{s}$. Further, the viscosities of mixtures of monoethanol amine and water mixtures under temperature 20-130 ${ }^{\circ} \mathrm{C}$ are measured. This is the normal temperature range for a traditional amine based $\mathrm{CO}_{2}$ capture process. Viscosity deviations are modeled according to Redlich-Kister type correlation and parameters are found through a regression analysis.
\end{abstract}

Index Terms - Viscosity, systematic error, uncertainty, MEA.

\section{INTRODUCTION}

Accurate measurement of viscosity in amine solutions is necessary for various aspects of gas treatment. Measurement of liquid viscosity is useful to determine flow behaviors and gas-liquid mass and heat transfer coefficients. Various viscometers are available to measure fluid viscosity with different measuring techniques. Capillary and coaxial cylinder type viscometers are widely used to measure fluid viscosity and coaxial cylinder viscometers are available in several geometries.

The uncertainty associated with viscosity measurements depends on many factors, which are engaged with the method that is used. Rheometer calibration provides valuable information about the systematic error of the instrument. The viscosity of a standard oil is measured and checked with the standard viscosities provided by the rheometer manufacturer to check for any kind of deviation between measured and standard viscosity. This difference is simply known as the error of the instrument and it can be further specified as a combination of both random and systematic errors in the instrument. Random errors are caused by unpredictable and unknown changes in the experiment. Changes can happen in

Manuscript received January 15, 2018; revised May 10, 2018.

The authors are with the University of Southeast Norway, Norway (e-mail: sumuduunimrt @gmail.com) the measuring instrument or environment conditions Gaussian distribution is often used to describe the nature of the random error. Systematic errors generally appear from the measuring instruments. In the instrument, systematic errors are present due to several reasons as poor calibration of the instrument, observational factors, environmental factors and use of simplified models and approximations. This error can be identified and reduced through carefulanalys is of the possible sources of systematic error.

Laun et al. [1] provided guidelines for checking performance and verifying the accuracy of rotational rheometers. Functional relations of viscosity with torque and angular speed for several types of rotational rheometers are also listed. Marvin [2] performed a study to investigate the systematic error of capillary type rheometer that was used for the viscosity measurement of water. Bringas et al. [3] discuss a calibration method for a new type of rheometer having a shaft with an uneven geometry. For the measurement uncertainty, Arachchige et al. [4] have calculated the combined uncertainty for the MCR 101 Anton Paar double-gap rheometer as $0.015 \mathrm{mPa} \cdot \mathrm{s}$ for the monoethanol amine (MEA) solutions. Hartono et al. [5] reported a value for the combined uncertainty for both $\mathrm{CO}_{2}$ loaded and unloaded MEA solutions as $0.007 \mathrm{mPa} \cdot \mathrm{s}$ for the Anton Paar MCR 100 rheometer. Amundsen et al. [6] estimated the measurement uncertainty of $0.01 \mathrm{mPa} \cdot \mathrm{s}$ for all viscosity measurements of MEA solutions.

In this study, a coaxial cylinder type rheometer placed in the $\mathrm{CO}_{2}$ laboratory in University college of Southeast Norway was examined to analyze the measurement errors during the viscosity measurements of mixtures of MEA and water. Experiments were performed to measure the viscosity of MEA, water mixture under MEA concentration from $20 \%$ to $50 \%$ by weight for the temperature range of $20{ }^{\circ} \mathrm{C}$ to $130{ }^{\circ} \mathrm{C}$. The uncertainty of the viscosity measurement was determined according to the guidelines provided by GUM (Guide to the expression of uncertainty in measurement) [7].

\section{METHOD}

\section{A. Random, Systematic Error of the Instrument}

Random and systematic errors of the instrument were examined through several viscosity measurements on a standard fluid. The viscosities of a standard fluid (calibration viscosities) were compared with measured viscosities of the same fluid through the rheometer under different temperatures as provided by the manufacturer. The viscosity of the standard fluid was measured several times to observe the consistency of the error. Further, measurement errors were predicted for the other temperatures through a regression 
analysis. The temperature range between $20{ }^{\circ} \mathrm{C}$ and $130{ }^{\circ} \mathrm{C}$ is the normal temperature range for a traditional amine based $\mathrm{CO}_{2}$ capture process. Predicted values were used to correct the viscosity measurements of MEA and water mixtures without $\mathrm{CO}_{2}$.

\section{B. Uncertainty of Viscosity Measurement}

The uncertainty of viscosity measurements signifies the quality of the measurement. It indirectly evaluates the measuring technique and is useful to improve the measurements. QUAM (Quantifying Uncertainty in Analytical Measurement) suggested some typical uncertainty sources that can be involved in a measuring process [8]. A cause and effect diagram is a graphical interpretation of uncertainty sources and it shows how individual uncertainties direct into a combined uncertainty. GUM provides guidance to evaluate the uncertainty of a measuring system [7].

The functional relationship between a measured quantity $\boldsymbol{x}=\left\{x_{i}\right\}$ (the input) and the measurement result $y$ (the output) can be shown as [7].

$$
\begin{aligned}
y & =f(x) \\
u^{2}(y) & =\sum_{i=1}^{n} \sum_{j=1}^{n} \frac{\partial f}{\partial x_{i}} \frac{\partial f}{\partial x_{j}} u\left(x_{i}, x_{j}\right)
\end{aligned}
$$

where, $\partial f / \partial x_{i}$ and $u\left(x_{i}, x_{j}\right)$ are the sensitivity coefficient and the covariance of $x_{i}$ and $x_{j}$ respectively. There, $u\left(x_{i}, x_{i}\right)=u^{2}\left(x_{i}\right)$ is the variance of $x_{i}$.

\section{Model for a Coaxial Cylinder Rheometer}

A mathematicalmodel is useful to understand the parameter that involves calculating the viscosity of the liquid in a coaxial cylinder rheometer. It is also helpful to identify the uncertainty sources in the uncertainty analysis. In the rheometer, there are two fluid compartments and the central cup rotates with a certain angular velocity. Fig. 1 shows the velocity profile of the liquids near the fixed and rotating boundaries.

Fluid behaviour can be illustrated by three differential equations in cylindrical coordinates [9].

$$
\begin{array}{r}
-\frac{\rho v_{\vartheta}^{2}}{r}=-\frac{\partial p}{\partial r} \\
-\frac{1}{r^{2}} \frac{\partial}{\partial r}\left[r^{2} \tau_{r \vartheta}\right]=0 \\
-\frac{\partial p}{\partial z}=\rho g
\end{array}
$$

where $\rho$ is density, $\tau$ is shear stress, $v$ is velocity and $r$ is radius. Consider $r=k R$

$R$ is the radius of inner fixed cylinder

\begin{tabular}{|c|c|c|c|}
\hline \multicolumn{2}{|c|}{ Inner region : $1<k<k_{1}$} & \multicolumn{2}{|c|}{ Outer region : $k_{2}<k<k_{3}$} \\
\hline $\begin{array}{l}\text { Boundary } \\
\text { condition }\end{array}$ & Velocity & $\begin{array}{l}\text { Boundary } \\
\text { condition }\end{array}$ & Velocity \\
\hline$r=R$ & $v_{\vartheta}=0$ & $r=k_{2} R$ & $v_{\vartheta}=\omega k_{2} R$ \\
\hline$r=k_{1} R$ & $v_{\vartheta}=\omega k_{1} R$ & $r=k_{3} R$ & $v_{\vartheta}=0$ \\
\hline
\end{tabular}

$k_{1}=\frac{R_{1}}{R}, k_{2}=\frac{R_{2}}{R}$ and $k_{3}=\frac{R_{3}}{R}$

Boundary conditions of the fluid flow in coaxial cylinder rheometer is summarized in Table I.
TABLE I: BOUNDARY CONDITION

Then, torque provided by the motor can be found as

$$
T=4 \pi \mu L \varpi R^{2}\left(\frac{k_{1}^{2}}{k_{1}^{2}-1}+\frac{k_{2}^{2} k_{3}^{2}}{k_{3}^{2}-k_{2}^{2}}\right)
$$

Here, $T$ is torque, $\mu$ is viscosity, $\mathrm{L}$ is the liquid height and $\omega$ is angular velocity

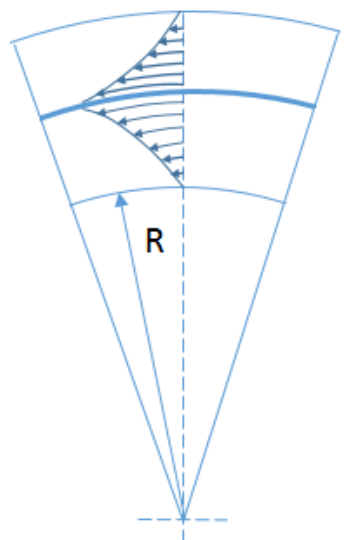

Fig. 1. Velocity profile of fluid in the coaxial cylinder.

Then the viscosity can be determined as shown in (7) and later it is used for the evaluation of measurement uncertainty of viscosity.

$$
\mu=\frac{T}{4 \pi L \varpi R^{2}\left(\frac{k_{1}^{2}}{k_{1}^{2}-1}+\frac{k_{2}^{2} k_{3}^{2}}{k_{3}^{2}-k_{2}^{2}}\right)}
$$

\section{Viscosity of MEA, Water Mixtures}

A series of experiments was performed to determine the vis cosity of MEA and water mixtures at a temperature range of $20{ }^{\circ} \mathrm{C}$ to $130{ }^{\circ} \mathrm{C}$. The MEA content of the mixture varied from $20 \%$ to $50 \%$ (wt \%). The calculated instrument error and uncertainty were used to present the measured data to enhance the accuracy of the measurements.

In experiments, the viscosity was measured using Physica MCR 101 rheometer supplied by Anton Paar. MEA with as say $\geq 99.5 \%$ provided by the ALDRICH was used for the sample preparation. Both MEA and water were degas sed using Buchi R-210 Rotavapor, evaporator to remove dissolved gases in the liquids. Liquids were weighed using an analytical balance, model XS403S from Mettler Toledo. A sample of $7 \mathrm{ml} \mathrm{MEA} \mathrm{/}$ water mixture was measured and transferred into the rheometer for measurements. The numbers in Table AI are average values of three original measurements. 


\section{RESULTS}

\section{A. Systematic Error}

The calibration fluid from Paragon scientific has been used to examine the measurement error of the instrument. Three calibration tests were performed at the beginning, middle and end of viscosity measurements of monoethanol amine and water mixtures. The results of the calibration test were compared with standard viscosities of calibration fluid to calculate instrument error at different temperatures. These experiments showed that the instrument error is not constant and it was concluded to perform several calibration tests to determine instrument error during the viscosity measurements of monoethanol amines. Fig. 2 shows the average values of the instrument error considering three different calibration tests.

In order to present the measured viscosities, a single value for the instrument error is needed to be calculated. The effective way to do it is to get the average value of instrument error for the measured temperature range. It also leads to eliminate the random error and systematic error will remain unchanged. Then, polynomial regression was performed on averaged errors to find a better relation to predicting instrument error for unknown temperatures. Fig. 2 shows the $4^{\text {th }}$ order polynomial fit for the instrument errors obtained through calibration tests.

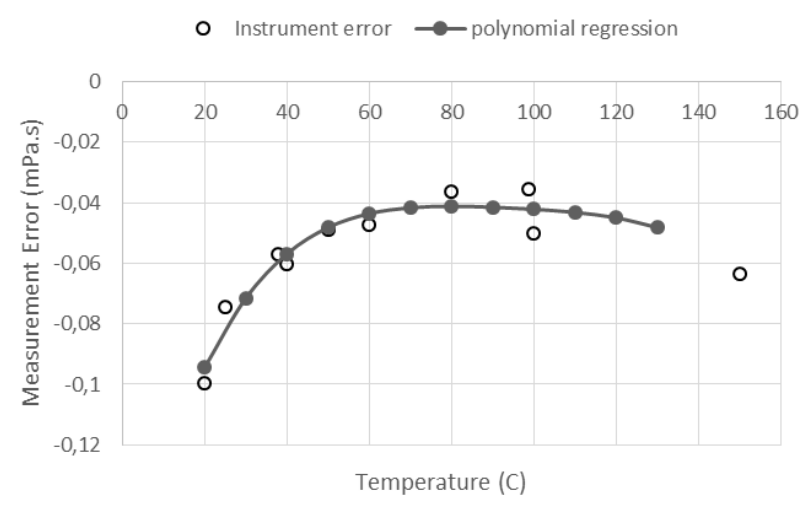

Fig. 2. Error in viscosity measurement of standard liquid.

Consequently, the measured viscosities of MEA and water mixtures were adjusted according to the evaluated instrument error.

Parameters of the mathematical model (8) for the calibration viscosities were obtained through a regression analysis to compare with the measured viscosities of standard fluid with predicted measurement errors for the temperature range of $20-130{ }^{\circ} \mathrm{C}$. The estimated values of the coefficients of (8) are shown in Table II.

$$
\ln (\mu)=a+\frac{b}{T-c}
$$

where, $\mu$ is viscosity ( $\mathrm{mPa} \cdot \mathrm{s}), T$ is temperature $(\mathrm{K})$ and a, b and c are coefficients [4].

T ABLE II: ESTIMATED PARAMETERS OF THE EQUATION

\begin{tabular}{|c|c|}
\hline Parameter & Values \\
\hline $\mathrm{a}$ & -3.298 \\
\hline
\end{tabular}

\begin{tabular}{|c|c|}
\hline $\mathrm{b}$ & 834.1 \\
\hline $\mathrm{c}$ & 112 \\
\hline
\end{tabular}

\section{B. Uncertainty}

The mathematical relation shown in (7) provides the relation between liquid viscosity with torque, angular speed, level of liquid fill and radius. In the experiments, a certain measured volume $(7 \mathrm{ml})$ of liquid is filled into the coaxial cylinder of the rheometer. The instrument provides the required torque to rotate the cup to maintain the shear rate at a desired level and the motor torque is measured. There are many uncertainty sources involved in viscosity measurements of amine solutions. A cause and effect diagram shown in Fig. 3 summarizes most of the uncertainty sources engaged in the experiment.

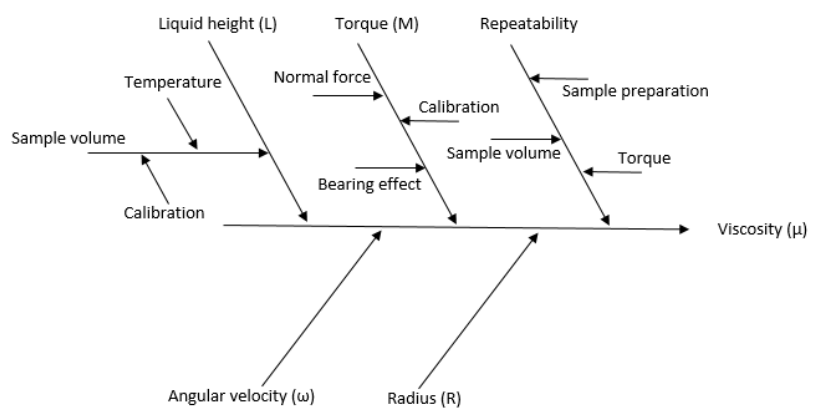

Fig. 3. Cause and effect diagram for uncertainties in viscosity measurements of a MEA / water mixture

The derived mathematical expression was used to identify the list of uncertainty sources. In addition to the parameters in the expression, there are some other parameters, which affect the measurement result but do not explicitly appear in the expression [8]. Those parameters are introduced as correction factors to the measurand. The following parameters have been identified as potential uncertainty sources but they are not included in the mathematical expression.

$$
\begin{gathered}
f_{p}: \text { Purity of MEA } \\
f_{t}: \text { Temperature } \\
f_{w}: \text { Weight measurement } \\
f_{\text {rep }}: \text { Repeatability }
\end{gathered}
$$

Then the expanded equation for viscosity measurement of the coaxial cylinder rheometer with considered correction factors can be shown as

$$
\mu=\frac{T}{4 \pi L \varpi R^{2}\left(\frac{k_{1}^{2}}{k_{1}^{2}-1}+\frac{k_{2}^{2} k_{3}^{2}}{k_{3}^{2}-k_{2}^{2}}\right)} f_{p} f_{t} f_{w} f_{\text {rep }}
$$

Combined uncertainty of the viscosity measurement was determined considering (9) as it covers most of the uncertainty sources in measuring. Kragten's approach [10] was used to estimate the sensitivity coefficient of combined uncertainty expression of (10). 


$$
u_{c}(\mu)=\sqrt{\left[\frac{\partial \mu}{\partial T} u(T)\right]^{2}+\left[\frac{\partial \mu}{\partial \omega} u(\omega)\right]^{2}+\left[\frac{\partial \mu}{\partial L} u(L)\right]^{2}+\left[\frac{\partial \mu}{\partial R} u(R)\right]^{2}+\left[\frac{\partial \mu}{\partial f_{p}} u\left(f_{p}\right)\right]^{2}+\left[\frac{\partial \mu}{\partial f_{t}} u\left(f_{t}\right)\right]^{2}+\left[\frac{\partial \mu}{\partial f_{w}} u\left(f_{w}\right)\right]^{2}+\left[\frac{\partial \mu}{\partial f_{\text {rep }}} u\left(f_{\text {rep }}\right)\right]^{2}}
$$

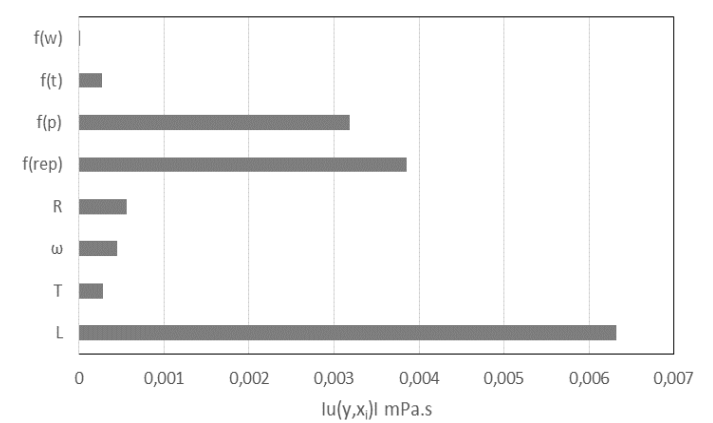

Fig. 4. Uncertainty contribution in viscosity measurements.

Fig. 4 shows the values obtained for the uncertainty sources. Thus, the calculated standard uncertainty of viscosity measurements in a coaxial cylinder rheometer is \pm $0.0081 \mathrm{mPa} \cdot \mathrm{s}$.

\section{Viscosity of MEA (1) +Water (2) Mixtures}

The corrected measured viscosities of aqueous MEA solutions from $20 \%$ to $50 \%$ of MEA in the temperature range of $20-130^{\circ} \mathrm{C}$ are shown in Fig. 5 and the values are listed in Table AI. The measured viscosities through rheometer were corrected according to the instrument error that was found in section III $A$. The calibration test results are shown in Table AII.

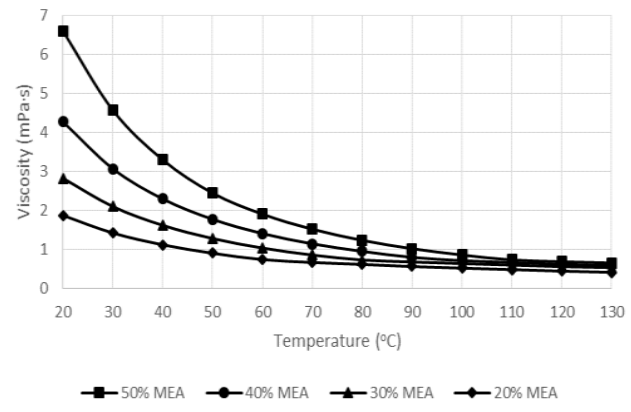

Fig. 5. Corrected viscosity vs Temperature in MEA and water mixtures.

The unloaded viscosity $\left(\mu_{\text {unloaded }}\right)$ can be presented as the sum of "ideal" viscosity based on the weighted sum of solution component's pure viscosities and a viscosity deviation $\left(\mu_{\gamma}\right)$.

$$
\ln \left(\mu_{\text {unloaded }}\right)=\ln \left(\mu_{Y}\right)+\sum_{1}^{2} x_{i} \ln \mu_{i}
$$

Hartono et al. [5] suggested a simplified Redlich-Kister [11] type correlation to model the viscosity deviation as a function of temperature and concentration.

$$
\ln \left(\mu_{\gamma}\right)=\left(a_{1}+a_{2} T+a_{3} T^{2}+a_{4} x_{1}\right) x_{1} x_{2}
$$

where, $\left(\mu_{i}\right)$ represents viscosity of the pure liquid, $x_{i}$ represents mole fraction and $T\left({ }^{\circ} \mathrm{C}\right)$ represents the temperature. The coefficients $a_{1}, a_{2}, a_{3}$ and $a_{4}$ are determined by a regression analysis on calculated viscosity deviation. The viscosities of pure solutions of MEA and water were obtained from Joseph et al. [12] and Udara et al. [4] respectively.

The calculated parameters for viscosity deviation are shown in Table III. The absolute average relative deviation (AARD) was calculated to examine the competence of model prediction and it was $6.2 \%$. The parameters found in this study are in the same order of magnitude compared to the Hartono et al. [5].

T ABLE III: PARAMETERS FOR THE VISCOSITY DEVIATION

\begin{tabular}{|c|c|l|}
\hline $\begin{array}{c}\text { MEA } \\
\text { (mass \%) }\end{array}$ & $\begin{array}{c}\text { Temperature } \\
\left({ }^{\circ} \mathrm{C}\right)\end{array}$ & $\begin{array}{l}\text { Parameters } \\
\text { (with 95\% confidence) }\end{array}$ \\
\hline 20 to 50 & 20 to 130 & $a_{1}[-]=10.23 \pm 1.075$ \\
& & $a_{2}\left[\left({ }^{\circ} \mathrm{C}\right)^{-1}\right]=-0.07747 \pm 0.02614$ \\
& & $a_{3}\left[\left({ }^{\circ} \mathrm{C}\right)^{-2}\right]=(4.351 \pm 1.708) .10^{-4}$ \\
& & $a_{4}[-]=-11.53 \pm 3.471$ \\
\hline
\end{tabular}

\section{DISCUSSION}

The analysis of instrument error in the rheometer enhance the accuracy of the final viscosity measurements. The method discussed in section III $A$ gives simple guidance to evaluate the random and systematic errors of rheometer in viscosity measurement of MEA solutions.

The AARD for the modeled and measured viscosities of the standard fluid was calculated to $0.7 \%$ for the measured temperature range of $20-130{ }^{\circ} \mathrm{C}$ under $10{ }^{\circ} \mathrm{C}$ degrees increment. The final viscosity values of MEA solutions obtained through this work were compared with data available in the literature. The viscosities obtained in this study showed a good agreement (difference $<2 \%$ ) with viscosity data presented by Hartono et al. [5] for low temperatures around $20{ }^{\circ} \mathrm{C}$. The measured viscosities by Hartono et al. [5], Arachchige et al. [4] and Amundsen et al. [6] were compared using the correlation developed in section III $C$. Table IV summarizes the AARD of the model for different sources.

T ABLE IV: AVERAGE AbSOlute Relative DeVIATION OF THE MODEL FOR MEA SOLUTIONS

\begin{tabular}{|l|c|l|c|}
\hline $\begin{array}{c}{[\mathrm{MEA}]} \\
(\mathrm{mass} \%)\end{array}$ & $\begin{array}{c}\text { Temperature } \\
\left({ }^{\circ} \mathrm{C}\right)\end{array}$ & $\begin{array}{c}\text { AARD } \\
(\%)\end{array}$ \\
\hline 30 & $20-80$ & Hartono et al. (2014) [5] & 4.2 \\
\hline $20-50$ & $20-80$ & Arachchige et al. (2013) [4] & 5 \\
\hline $20-50$ & $25-80$ & Amundsen et al. (2009) [6] & 4.2 \\
\hline $20-50$ & $20-130$ & This work & 6.2 \\
\hline
\end{tabular}

The AARD was found to be $4.2 \%$ for the viscosity predictions using this correlation and measured viscosity by Hartono et al. [5]. It is similar to the AARD of literature data and predictions from the original correlation proposed by Hartono et al. [5]. The AARD found in this study for the temperature range of $\left(20-130{ }^{\circ} \mathrm{C}\right)$ is higher $(6.2 \%)$. This is probably due to the higher temperature levels that the 
viscosities were measured. Amundsen et al. [6] have stated that the higher temperatures increase the uncertainty.

The reported uncertainties for the viscosity measurement were compared with the uncertainty obtained through this study. The uncertainty calculated in this work showed a higher value compared to the reported measurement uncertainties in section I. The method discussed in section III $B$ considered most of the possible uncertainty sources involved in the measurement method, which resulted in a higher value of combined uncertainty.

Currently, various researches have given attention to measuring physicochemical properties of different amine solutions. As a continuation of this study, the viscosity of $\mathrm{CO}_{2}$ loaded MEA and other different amines will be measured and correlated to extend the range of measurement. The uncertainty of viscosity measurements in $\mathrm{CO}_{2}$ loaded aqueous amine solutions also will be determined and validated using Monte Carlo simulation (MCS) method.

\section{CONCLUSION}

Both randomand systematic errors have influenced on final viscosity measurements of MEA and water mixtures in the rheometer. Viscosity measurements on calibration fluid can be used to estimate the systematic error of the instrument. Random effects of the measurement error are reduced by taking multiple viscosity measurements on the calibration fluid.

A mathematical expression was obtained relating parameters involved in the viscosity measurements in the coaxial cylinder rheometer. It is useful to identify the uncertainty sources involved in the measurement method. The calculated expanded uncertainty $(\mathrm{k}=2)$ for the coaxial cylinder rheometer is $0.0162 \mathrm{mPa} \cdot \mathrm{s}$.

The viscosity of MEA and water mixtures increases with the rise of MEA content in the mixture. In addition to that, the viscosity of MEA and water mixtures gradually decreased when the temperature of the mixture increased. The developed model for viscosity deviation was able to predict measured data with an AARD within $6.2 \%$.

\section{APPENDIX A}

T ABLE AI: CORRECTED VISCOSITY MEASUREMENTS OF MEA AND WATER MIXTURES

\begin{tabular}{|c|r|r|r|r|}
\hline \multirow{2}{*}{ Temperature $\left({ }^{\circ} \mathrm{C}\right)$} & \multicolumn{4}{|c|}{ Viscosity (mPa.s) at different MEA wt \% } \\
\cline { 2 - 5 } & $20 \%$ & $30 \%$ & $40 \%$ & $50 \%$ \\
\hline 20 & 1.8819 & 2.8359 & 4.2851 & 6.6100 \\
\hline 30 & 1.4308 & 2.1094 & 3.0801 & 4.5803 \\
\hline 40 & 1.1286 & 1.6279 & 2.3052 & 3.3093 \\
\hline 50 & 0.9122 & 1.2897 & 1.7821 & 2.4543 \\
\hline 60 & 0.7503 & 1.0456 & 1.4168 & 1.9145 \\
\hline 70 & 0.6749 & 0.8660 & 1.1541 & 1.5280 \\
\hline 80 & 0.6252 & 0.7398 & 0.9604 & 1.2430 \\
\hline 90 & 0.5697 & 0.6872 & 0.8077 & 1.0291 \\
\hline 100 & 0.5294 & 0.6481 & 0.7232 & 0.8676 \\
\hline
\end{tabular}

\begin{tabular}{|l|l|l|l|l|}
\hline 110 & 0.4919 & 0.6088 & 0.6742 & 0.7408 \\
\hline 120 & 0.4521 & 0.5693 & 0.6285 & 0.6943 \\
\hline 130 & 0.4226 & 0.5423 & 0.5991 & 0.6624 \\
\hline
\end{tabular}

Uncert ainty: $U(\mu)= \pm 0.0162 \mathrm{mPa} \cdot \mathrm{s}$ (Level of confidence $=0.95$ where $\mathrm{k}=2$ )

T ABLE AII: VISCOSITY OF THE STANDARD FLUID

\begin{tabular}{|c|c|c|}
\hline $\begin{array}{c}\text { Temperature } \\
\left({ }^{\circ} \mathrm{C}\right)\end{array}$ & $\begin{array}{c}\text { Viscosity of Standard fluid } \\
(\mathrm{mPa} \cdot \mathrm{s})\end{array}$ & $\begin{array}{c}\text { Measured Viscosity } \\
\text { of Standard fluid } \\
(\mathrm{mPa} \cdot \mathrm{s})\end{array}$ \\
\hline 20 & 3.714 & 3.614247 \\
\hline 25 & 3.267 & 3.192492 \\
\hline 37.78 & 2.439 & 2.382142 \\
\hline 40 & 2.327 & 2.266567 \\
\hline 50 & 1.913 & 1.864058 \\
\hline 60 & 1.603 & 1.555717 \\
\hline 80 & 1.177 & 1.140617 \\
\hline 98.89 & 0.918 & 0.882373 \\
\hline 100 & 0.9065 & 0.856242 \\
\hline 150 & 0.5365 & 0.472868 \\
\hline
\end{tabular}

\section{REFERENCES}

[1] M. Laun et al., "Guidelines for checking performance and verifying accuracy of rotational rheometers: Viscosity measurements in steady and oscillatory shear (IUPAC Technical Report)," Pure Appl. Chem., vol. 86, no. 12, pp. 1945-1968, 2014.

[2] R. S. Marvin, "The accuracy of measurements of viscosity of liquids," Journal Of Research at the National Bureau of Standards, vol. 75A, no. 6, pp. 535-540, 1971.

[3] C. S. Bringas, W. K. Jeksrud, O. I. Lekang, and R. B. Schuller, "A calibration method for a new type of rheometer," Annual Transactions of the Nordic Rheology Society, vol. 14, 2006.

[4] U. S. P. R. Arachchige, N. Aryal, D. A. Eimer, and M. C. Melaaen, "Viscosities of pure and aqueous solutions of monoethanolamine (MEA), diethanolamine (DEA) and N-Methyldiethanolamine (MDEA)," Annual Transactions of the Nordic Rheology Society vol. 21, 2013.

[5] A. Hartono, E. O. Mba, and H. Svendsen, "Physical properties of partially $\mathrm{CO} 2$ loaded aqueous monoethanolamine (MEA)," $J$. Chem. Eng, vol. 59, pp. 1808-1816, 2014.

[6] T. G. Amundsen, L. E. Øi, and D. A. Eimer, "Density and viscosity of monoethanolamine + water + carbon dioxide from (25 to 80) C," J.Chem.Eng.Data, vol. 54, pp. 3096-3100, 2009.

[7] "Evaluation of measurement data - Guide to the expression of uncertainty in measurement," 2008.

[8] S. L. R. Ellison and A. Williams, "Quantifying uncertainty in analytical measurement," 2012.

[9] R. B. Bird, W. E. Stewart, and E. N. Lightfoot, Transport Phenomena, John Wiley \& Sons, Inc, 2002.

[10] I. Leito, L. Jalukse, and I. Helm, "Estimation of measurement uncertainty in chemical analysis (analytical chemistry) course."

[11] O. Redlich and A. T. Kister, "Algebraic representation of thermodynamic properties and the classification of solutions," Industrial and Engineering Chemistry, vol. 40, no. 2, pp. 345-348, 1948.

[12] J. Kestin, M. Sokolov, and W. A. Wakeham, "Viscosity of liquid water in the range -8 C to 150 C," J. Phys. Chem. Ref. Data, vol. 7 , no. 3, pp. 941-948, 1978.

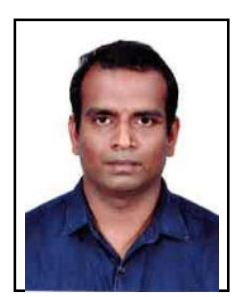

Sumudu S. Karunarathne received his second MSc degree in process technology (University of Southeast Norway (USN), Porsgrunn, Norway, 2016) and first MSc degree in sustainable process development (University of Moratuwa (UOM), Moratuwa, Sri Lanka, 2012) after a BSc in chemical and process engineering (UOM, 2008).

He is a PhD student at USN since 2016, where his 
topic is related to measurement of phy sicochemical data for amine based $\mathrm{CO}_{2}$ capture process. He has worked as a lecturer in Institute of Technology, University of Moratuwa (ITUM) (2011-2016), and as a chemical engineer in Aqua Technologies (Pvt) Ltd, Sri Lanka.

Sumudu's research interests are post combustion $\mathrm{CO}_{2}$ capture, modelling and simulation, powder technology and water and wastewater treatment.

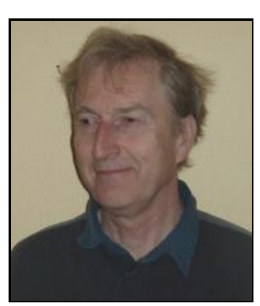

Dag Fimer has a $\mathrm{PhD}$ in chemical engineering from NTNU, Trondheim, Norway (1994), and a B.Sc. in chemical enigeering from University of Strathclyde, Glasgow, Scotland (1970). He is a professor of chemical engineering in the University of South-Eastern Norway since 2008 where he is engaged in $\mathrm{CO}_{2}$ capture research. $\mathrm{He}$ was previously at Norsk Hydro Research Centre, and later Norsk Hydro Oil \& Energy for 37 years, and is the author on the book Gas Treating - Absorption theory and practice, published by Wiley in 2014 .

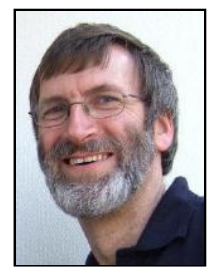

Lars Erik Øi is a professor in process technology at University of Southeast Norway. He received his master in chemical engineering from NTH in Trondheim in 1983 and his $\mathrm{PhD}$ in process, energy and automation engineering from Telemark University College in 2012.

He has worked 8 years as a researcher for the company Norsk Hydro and 23 years as an associate professor and full professor at University College of Southeast Norway (earlier Telemark University College). He is lecturing courses in Thermodynamics and Gas purification and energy optimization. His research fields are process technology and separation technology in general, and glycol dehydration and $\mathrm{CO}_{2}$ capture in special. 